\title{
Un programa de seguimiento comunitario de personas con enfermedad mental
}

RESUMEN: Descripción de la intervención realizada por la FISLEM; se presentan datos de la muestra estudiada y de los objetivos, metodología y desarrollo del estudio.

PALABRAS CLAVES: Programa comunitario, enfermedad mental, rehabilitación psicosocial.
SUMMARY: We show a description of intervention achieved by the FISLEM and display some data of the sample, objectives, methodology and study development.

KEY WORDS: Community care program, mental illness, psychosocial rehabilitation.

En el año 2000 se inicia el movimiento más importante de creación de recursos de salud mental comunitarios en Castilla-La Mancha. En ese momento el número de pacientes internados en la Unidades de Larga Estancia de las Diputaciones Provinciales (URR) era de 522 (1), y el número de pacientes atendidos en recursos comunitarios de rehabilitación psicosocial, laboral y viviendas supervisadas era de 332 (2). Seis años más tarde son unos 200 pacientes los que permanecen en unidades de larga estancia (un 38\% menos), así como 2.500 los que son atendidos desde recursos comunitarios de rehabilitación y apoyo comunitario (750\% más). Estas cifras demuestran que los programas de rehabilitación se han constituido en un elemento esencial en la atención a la población con enfermedades mentales de larga evolución en la comunidad. Sin embargo este crecimiento en recursos y plazas ha venido condicionado por factores de los propios recursos de atención y de las características de la propia comunidad, que originaron pronto nuevas necesidades en el proceso de reforma. El principal de ellos fue el evidenciar la necesidad de programas específicos que requieren la continuidad de cuidados y la interrelación entre distintos servicios para atender a los pacientes crónicos más desvinculados de la atención y con mayores dificultades para acceder a los recursos sociosanitarios. Otros factores que también han condicionado el desarrollo de los programas de rehabilitación psicosocial en Castilla-La Mancha ha sido el retraso en la dotación de este tipo de recursos, el entorno sociodemográfico rural disperso y las propias necesidades del trastorno mental grave (escaso uso de redes sociosanitarias, discapacidades añadidas, escasa red de apoyo, etc.). Aunque el sistema público ha favorecido la creación y especialización de servicios para el trastorno mental grave (TMG), los factores anteriores llevan a considerar algunos aspectos que han afectado a la planificación. Los nuevos centros de rehabilitación ubicados en la comunidad y las alternativas residenciales se orientaron inicialmente hacia las personas que ya estaban localizadas en ese momento, que vivían en la comunidad y acudían a las revisiones clínicas con un 
terapeuta de referencia, o que eran dados de alta tras un ingreso breve o de una unidad de larga estancia (en definitiva, los pacientes que ya estaban localizados por el sistema). Para el año 2003 era patente que aún en los lugares en los que los recursos habían servido para cubrir las necesidades de la población un grupo de pacientes muy necesitados de ellos podría no utilizarlos debido tanto a su trastorno, dificultad para hacer explícitas sus demandas (3), como al contexto, que facilitaba la dificultad de acceso en algunos ámbitos rurales y alejados. Se empieza a ver que algunas personas con TMG, especialmente esquizofrenia, carecían de iniciativas para recibir atención en programas de rehabilitación psicosocial, incapaces de gestionarse la «nueva ayuda» o difícil de vincularlos a los nuevos recursos comunitarios del área. Según esto, un porcentaje alto de personas con TMG permanecerían en riesgo de exclusión social, con dificultades de accesibilidad y de abordar la compleja interacción entre el contexto y el diseño de recursos (las estimaciones iniciales estimaban que entre el $30 \%$ y el $40 \%$ del total de personas TMG estarían en esa situación). Se puso de manifiesto la necesidad de desarrollar programas que resolvieran ese problema. El principal reto era la adaptación de programas de rehabilitación psicosocial tradicionales a la realidad histórica y actual de Castilla-La Mancha (4). Así, se puso en marcha el Programa Regional de Integración Social (PRIS) de personas con enfermedad mental (5), reconociendo la situación de desventaja de ese tipo de paciente y su familia, y asumiendo la responsabilidad de hacer llegar la atención en rehabilitación psicosocial allí donde no llegaba. El programa se extendió desde el año 2002 al 2004. En el año 2005 se convierte en un programa integrado en los Centros de Rehabilitación Psicosocial y Laboral (CRPSL). En este trabajo se describe el desarrollo y las características de este programa.

\section{Programas de seguimiento en la comunidad}

Ya desde los años setenta, ha quedado patente que en los lugares donde se han desarrollado recursos puede suceder que una población necesitada de ellos no los utilice debido a su trastorno o a variables relacionadas con la red de atención (6). En respuesta a esas dificultades se han desarrollado distintos modelos con diferentes denominaciones: programas de seguimiento comunitario, de continuidad de cuidados, de atención en el domicilio, etc. La función de estos programas es evaluar las necesidades del paciente, desarrollar un plan de atención individualizado, la coordinación de los servicios y el seguimiento continuo en la red. Estos programas tienen cinco funciones: evaluación, planificación, coordinación, seguimiento y defensa de los intereses de los pacientes (7). Algunos de los principios 
esenciales de la atención ya fueron recogidos por Barcharch en 1993 (8), aquí destacamos algunos de ellos: accesibilidad, servicios integrales (desde el tratamiento psiquiátrico hasta las actividades de ocio), tratamiento individualizado, flexibilidad (en espacios y tiempos), coordinación entre dispositivos y pertinencia cultural (adaptados a la realidad del paciente).

Existe una gran variedad en su organización, selección de tareas que llevan a cabo en la atención que prestan a los pacientes y las funciones asignadas al profesional de referencia. Hay varias revisiones globales de los distintos modelos de seguimiento que sintetizan sus objetivos, características y resultados, como son las de Alonso, Bravo y Fernández (6), González (7) o Hernández (3). La agrupación de Mueser de 1998 (9) ha sido la más citada por ser la más útil a seguir con tablas muy didácticas para la comparación entre programas. Se proponen seis modelos: 1) modelo de agencia de servicios; 2) modelo de casemanagment clínico; 3) modelo de case-managment intensivo; 4) tratamiento asertivo-comunitario; 5) modelo de competencias o capacidades; 6) modelo rehabilitador. Se remite a cualquiera de las referencias para una revisión en detalle de cada uno de ellos.

Son muchos los estudios de eficacia y efectividad que arrojan evidencias a favor de los modelos de seguimiento. Los estudios se han realizado comparando la intervención con la gestión de caso estándar. La mayoría de los estudios incluyen pacientes con una historia de alta utilización de servicios, con deterioro funcional severo, que viven en la comunidad y presentan altas dificultades de vinculación al tratamiento. Los hallazgos más repetidos son la reducción de abandonos, el número de rehospitalizaciones y el tiempo de permanencia en el hospital, así como la satisfacción con la atención (10-12). En esa línea, los beneficios de los programas asertivo comunitarios cuentan con una considerable evidencia empírica que consiste en la mayor estabilidad en la vivienda, reducción del tiempo pasado en el hospital, calidad de vida y satisfacción $(9 ; 13 ; 14)$. La reducción en la severidad de los síntomas, de los costes económicos y la mejora en la integración social y laboral son resultados menos replicables, aunque se han encontrado hallazgos positivos cuando los programas estaban específicamente orientados a esos objetivos (15-17).

\section{Recomendaciones para los programas de seguimiento en la comunidad}

La continuidad de cuidados para los pacientes más graves es una de las principales aspiraciones en una atención comunitaria de calidad. Con el objetivo de asegurar este ideal se han realizado múltiples recomendaciones sobre 
cómo conseguir un funcionamiento de los servicios que haga esto posible. El tema es objeto de congresos, revisiones sistemáticas y publicaciones periódicas que tratan de definir y evaluar los componentes «ideales» o «facilitadores» que aseguren el desarrollo de programas de seguimiento. En esta línea, y siguiendo el análisis de Maite San Emeterio (18), se podrían enumerar una serie de factores relacionados con la planificación, con los servicios y profesionales, y con los pacientes y las familias. Entre otros incluye: disponer de normas de funcionamiento consensuadas y formas de acceso y alta, reuniones periódicas para evaluar los casos y resolver los problemas, canales ágiles de información entre los servicios, claridad en las figuras de referencia para el paciente, flexibilidad para la atención en el domicilio y énfasis en programas de rehabilitación psicosocial en todos los recursos.

Suárez, Bravo y Fernández (6), exponiendo los resultados cualitativos del proyecto IPSE en una muestra de pacientes con TMG, incluyen algunas recomendaciones que facilitan la implantación de programas de continuidad de cuidados: equipos pequeños, tener implantados procedimientos de detección, actitud activa del profesional en la fase de enganche, trabajar con los recursos de la comunidad de forma habitual, formación necesaria del profesional para proveer cuidados directamente, contacto continuado con el paciente, reuniones de equipo para discutir casos, incluir el trabajo con la familia, que exista una historia integrada en el servicio de salud mental de referencia y con la documentación de los seguimientos actualizada. El estudio PRISM (19) realizó varias revisiones sobre la eficacia de los servicios comunitarios de salud mental, generando varios artículos y editoriales sobre el tema (20-22). Los resultados muestran datos favorables en todas las formas de tratamiento comunitario en comparación con la atención psiquiátrica tradicional. Entre las recomendaciones que se extraen del grupo de investigación están: atención planificada por sectores o áreas geográficas, visitas frecuentes en el domicilio, el equipo debe ser multidisciplinar, existe una figura profesional de referencia para cada paciente con TMG de la red, atención rápida a las crisis agudas, y distintas fórmulas de equipos especializados en el seguimiento en la comunidad integrados en servicios tradicionales de salud mental.

Por otro lado, y siguiendo algunas recomendaciones para el diseño de programas de seguimiento de salud mental ajustados a contextos rurales y que cobran especial relevancia en nuestra Comunidad Autónoma, algunos facilitadores serían (4; 23): contacto simultáneo con el paciente y la familia en el domicilio, utilización de recursos no profesionales, facilidad para saltarse protocolos tradicionales en situaciones de urgencia, intervención con servicios de atención primaria de servicios sociales y de salud. 
DEBATES E INFORMES

\section{Dificultades para implantar programas de seguimiento en la comunidad}

Además del esfuerzo por definir claramente los elementos que facilitan la puesta en marcha de los programas, también se han identificado los obstáculos comunes, que van desde la diversidad en los programas desarrollados, hasta las habilidades y formación del equipo. En la experiencia anterior comentada del estudio IPSE (6) agrupan los obstáculos en cuatro: a) falta de medios (lo que conlleva la reducción de la intensidad y frecuencia del seguimiento de cada caso, falta de tiempo para la coordinación, acumulación de pacientes y exceso de áreas de intervención para cada profesional de referencia); b) la actitud del equipo (compartir la filosofía del trabajo, capacidad para trabajar en equipo, apoyo del director del servicio donde se integre el programa, colaboración de los centros socio-sanitarios implicados); c) la formación del equipo (para la selección de los pacientes, la evaluación y la planificación de la intervención); d) caer en la rutina (exceso de tareas administrativas, dejar de registrar los seguimientos y la evolución del paciente en la historia, dejar de hacer reuniones de equipo para discutir los casos). La principal dificultad apunta a la variación en la implantación de los programas, que hace difícil comparar resultados o replicar métodos de trabajo. También hay que mencionar la limitación de extrapolar a la práctica habitual gran parte de los programas publicados en forma de estudios experimentales.

Otro aspecto comentado antes en relación a los contextos rurales son los obstáculos específicos en el desarrollo de programas en estos contextos $(4 ; 23 ; 25$ 26): los profesionales tienen que recorrer largas distancias, la dificultad para atender rápido a la urgencia, la inversión de tiempo asociada a la atención en relación al bajo número de pacientes atendidos, la desconexión entre el programa y los servicios de salud mental centralizados en núcleos poblacionales más grandes, la escasa disponibilidad de alternativas comunitarias en zonas rurales pequeñas y empobrecidas y la falta de disponibilidad de profesionales facultativos para atender en el domicilio a los casos de los pueblos más alejados.

\section{Definición del programa PRIS y del contexto de la intervención}

Bajo la denominación «Programa de Integración Social y Apoyo Comunitario de personas con enfermedad mental» (PRIS), hemos desarrollado un programa con el objetivo de cubrir las necesidades de seguimiento y atención de un grupo de pacientes que tienen dificultades para acceder o beneficiarse de los servicios rehabilitadores (CRPSL de la comunidad) debido a causas que van desde la inaccesibilidad o la falta de motivación, hasta la carencia de soportes sociales. 
No solo por los resultados obtenidos por los programas de seguimiento sino también por el hecho de que constituyen una metodología para organizar la intervención y un vehículo a través del cual se facilitan los tratamientos, nos propusimos la creación de un programa de estas características. Por otro lado, el propio desarrollo de nuestra Comunidad Autónoma apuntaba a la idea de que podría construir un instrumento útil para el correcto aprovechamiento de una recién creada y compleja red de atención y rehabilitación, sobre todo para un tipo de paciente con mayores deterioros. Haciendo una revisión de los distintos modelos de programas parece claro que no hay un modelo igualmente apropiado para cualquier marco de atención. Esto plantea un debate continuo en la investigación en relación a poder demostrar la superioridad de unos modelos de programas de seguimiento frente a otros, o a la dificultad para encontrar los elementos clave o factores responsables del éxito del programa $(6 ; 19)$. Pese a esto, optamos por un programa que recogiera algunos de los elementos que son propios de este tipo de programas y que se adaptara a las características de nuestros servicios, nuestros equipos y nuestros pacientes, y en el momento de cambio organizacional en nuestra comunidad. Estamos lejos de querer fomentar el debate, y de defender la preferencia por uno u otro modelo. Nuestro objetivo es describir nuestra experiencia con un programa de seguimiento, específico y propio de nuestro contexto y posibilidades, eso sí, sin perder de vista los hallazgos de la investigación acerca de los componentes que parecen relacionarse con los mejores resultados. Para poder comprender el diseño del programa también es necesario describir el tipo de servicios en el que se integra, que también condiciona en gran medida las características de la intervención.

\section{Programa PRIS: definición y objetivos}

El programa fue desarrollado en el año 2002, en respuesta las necesidades de las personas con especiales dificultades de llegar la los CRPSL, asumiendo el papel de hacer llegar la atención en rehabilitación psicosocial en aquellos lugares de Castilla-La Mancha donde no llegaba. Aunque ha tenido diversos objetivos durante su implantación (ver tabla 1), el programa buscaba complementar la atención tradicional de los recursos que se iban creando, mediante la creación de un sistema de apoyo que ayudara y acompañara en la solución de los problemas de vida diaria. El programa responde a las necesidades del contexto en el que se mueven los pacientes y sus familias, un contexto caracterizado por la dispersión geográfica y las dificultades de accesibilidad, elementos que facilitan que los pacientes más graves se pierdan en la red o permanezcan «invisibles» para el sistema. Entre las líneas de actuación del PRIS ha estado por tanto la labor de complementar a los CRPSL en la atención a las zonas más rurales y 
Tabla1

Fases del programa PRIS de personas con TMG

\begin{tabular}{|c|c|c|c|}
\hline FASE & AÑO & OBJETIVO & RESULTADO \\
\hline $\begin{array}{l}\text { Creación del } \\
\text { programa y } \\
\text { detección de } \\
\text { necesidades }\end{array}$ & $2002-2003$ & $\begin{array}{l}\text { - Captación y detección de enfer- } \\
\text { mos mentales graves alejados de la } \\
\text { red de atención o que han perdido } \\
\text { su vinculación con los servicios de } \\
\text { salud mental y con altas necesida- } \\
\text { des de programas de rehabilitación } \\
\text { psicosocial } \\
\text { - Presentación y difusión del pro- } \\
\text { grama en los servicios y puertas de } \\
\text { entrada de la atención: atención } \\
\text { primaria, servicios sociales y uni- } \\
\text { dades de salud mental } \\
\text { - Garantizar el acceso a recursos de } \\
\text { rehabilitación psicosocial y laboral }\end{array}$ & $\begin{array}{l}\text { - Un total de } 899 \text { casos fue- } \\
\text { ron detectados, pasando a } \\
\text { fase de intervención } 519 . \\
\text { - Un total de } 227 \text { casos } \\
\text { entran a los CRPSL para } \\
\text { recibir programas indivi- } \\
\text { duales de rehabilitación }\end{array}$ \\
\hline
\end{tabular}

Integración del programa en los CRPSL
2004

- Especialización de funciones de apoyo a la rehabilitación psicosocial

- Estudio de casos y canalización de otros perfiles hacia recursos adecuados

- Inclusión como un subprograma de los CRPSL
- Reubicación territorial de los profesionales en los CRPSL

- 366 casos fueron evaluados y canalizados a otros recursos más adecuados, en coordinación con servicios sociales y salud mental

- Definición del programa con un diseño ajustado al perfil de TMG, incluyendo áreas de intervención y proceso de atención integrado en los CRPSL

\begin{tabular}{|c|c|c|c|}
\hline $\begin{array}{l}\text { Atención } \\
\text { comunitaria } \\
\text { continuada }\end{array}$ & 2004-2007 & $\begin{array}{l}\text { - Apoyo a la rehabilitación psicoso- } \\
\text { cial y laboral en zonas de influen- } \\
\text { cia del CRPSL } \\
\text { - Atención en el domicilio } \\
\text { - Apoyo social y acompañamiento } \\
\text { en casos de situación de aislamien- } \\
\text { to } \\
\text { - Enganche de casos que se desvin- } \\
\text { culan de la atención }\end{array}$ & $\begin{array}{l}\text { - Continuidad de cuidados } \\
\text { - Prevención de abandonos } \\
\text { y pérdida de casos } \\
\text { - Complemento de la aten- } \\
\text { ción comunitaria en zonas } \\
\text { rurales y alejadas } \\
\text { - } 300 \text { pacientes atendidos } \\
\text { como promedio anual }\end{array}$ \\
\hline
\end{tabular}


alejadas de los recursos, para un tipo de paciente en situación de riesgo social, aislamiento, tendencia a la cronificación y a la desvinculación del sistema de atención tradicional de salud mental. Desde el programa se ayuda al paciente en las demandas reales de su vida, se le acompaña en la solución de los problemas prácticos y se le facilita la adquisición de habilidades de autonomía. También se trabaja para ayudar a reforzar las relaciones con su entorno y para facilitar su participación en actividades comunitarias. Asuntos como la organización doméstica, acompañamientos a trámites, apoyo familiar, búsqueda de recursos económicos o servir de enlace con la red de apoyo informal son ejemplos de intervenciones concretas. Los objetivos específicos del programa son: 1) desarrollar un sistema de cuidados y asesoramiento de usuarios y familiares, en colaboración con los dispositivos y recursos de rehabilitación psicosocial y laboral; 2) elaborar programas individuales de seguimiento, apoyo y acompañamiento; 3) proporcionar a los usuarios acceso a los Servicios Sociales y Servicios de Salud Mental así como información sobre los recursos disponibles en su entorno; 4) mantener en la comunidad con criterios de normalización e integración social y laboral; 5) complementar los programas de rehabilitación y apoyo comunitario en aquellos lugares donde no llegan.

\section{Características de la intervención}

Por las características del programa, su estilo de intervención comparte algunos de los elementos de los programas denominados como case-managment, seguimiento o apoyo comunitario. En la tabla 2 se hace una comparativa del programa PRIS con esos otros programas de seguimiento. De los programas de case-managment más tradicionales recoge la tarea de ayudar al paciente a desenvolverse en la red de salud mental comunitaria. Un profesional de referencia es asignado a cada caso para evaluar las necesidades, evitar la desconexión con los servicios de salud mental y mantener un asesoramiento continuado. La intervención la realiza 1 profesional denominado Técnico de Inserción Socio-laboral integrado en el equipo multidisciplinar de un CRPSL (psicólogo, trabajador social, terapeuta ocupacional y monitor). Aunque el profesional es la referencia para el caso, el resto del equipo participa activamente en la toma de decisiones. El $75 \%$ de su trabajo son intervenciones en el domicilio, siendo el 25\% restante tiempo para reuniones de coordinación y planificación de actividades. Cada profesional del programa tiene asignados entre 15 y 20 casos. El trabajo que realizan se centra en ayuda práctica de la vida diaria, como parte de un plan individual de rehabilitación y seguimiento coordinado por el CRPSL y la Unidad de Salud Mental 
(USM). Las actividades incluyen tres grandes áreas de intervención: a) apoyo al proceso de rehabilitación psicosocial y laboral; b) apoyo social e integración comunitaria; c) apoyo en el domicilio. Otras líneas de actuación son la información y apoyo a la familia y al entorno social del paciente. Los profesionales tienen una actitud «activa» evitando la pérdida de casos y activando la visita en el domicilio cuando exista riesgo de abandono. El equipo está formado por 16 profesionales repartidos en 13 CRPSL, que dan atención a una media de 300 pacientes al año, en su mayoría adultos con esquizofrenia. La cobertura es de día, de lunes a viernes entre las 8 y las 19 horas.

Tabla 2

Comparación del programa PRIS con otros programas de seguimiento

\begin{tabular}{lcccc}
\hline \multicolumn{1}{c}{ Características } & $\begin{array}{c}\text { Modelo } \\
\text { case-management }\end{array}$ & $\begin{array}{c}\text { Modelo } \\
\text { rehabilitador }\end{array}$ & $\begin{array}{c}\text { Modelo } \\
\text { asertivo-comunitario }\end{array}$ & Programa PRIS \\
\hline $\begin{array}{l}\text { Proporción } \\
\text { profesional: } \\
\text { paciente }\end{array}$ & $1: 50$ & $1: 20-30$ & $1: 10$ & $1: 15-20$ \\
\hline $\begin{array}{l}\text { Casos compartidos } \\
\text { en equipo }\end{array}$ & No & No & Sí & Sí \\
\hline $\begin{array}{l}\text { Disponibilidad } \\
\text { del equipo }\end{array}$ & Limitada & Limitada & 24 horas & Limitada \\
\hline $\begin{array}{l}\text { Frecuencia } \\
\text { de contacto }\end{array}$ & Media/baja & Media & Alta & Media/alta \\
\hline $\begin{array}{l}\text { Entrenamiento } \\
\text { en habilidades }\end{array}$ & Bajo & Alto & Moderado & Alto \\
\hline $\begin{array}{l}\text { Lugar de } \\
\text { tratamiento }\end{array}$ & Consulta & $\begin{array}{c}\text { Consulta/ } \\
\text { comunidad }\end{array}$ & Comunidad & Comunidad \\
\hline $\begin{array}{l}\text { Provisión directa } \\
\text { del tratamiento }\end{array}$ & No & Parcial & Completo & $\begin{array}{c}\text { Coordinado servicios } \\
\text { de salud mental }\end{array}$ \\
\hline \begin{tabular}{l} 
Población diana \\
\hline $\begin{array}{l}\text { Contacto con } \\
\text { la familia }\end{array}$
\end{tabular} & EMG & EMG & EMGACR & EMGDAV \\
\hline $\begin{array}{l}\text { Uso de recursos } \\
\text { comunitarios }\end{array}$ & Bajo & Medio & Alto & Medio-Alto \\
\hline $\begin{array}{l}\text { Seguimiento } \\
\text { continuado }\end{array}$ & Alto & Medio & Alto & Alto \\
\hline
\end{tabular}

EMG: Enfermo mental grave.

EMGACR: Enfermo mental grave con alto consumo de recursos.

EMGDAV: Enfermo mental grave dificultades de accesibilidad y vinculación. 


\section{Contexto de la intervención: la Fundación FISLEM}

En Castilla-La Mancha se creó en el año 2001 la Fundación Socio-Sanitaria para la Integración Socio-Laboral del Enfermo Mental (FISLEM) con el objetivo entre otros de poner en marcha, coordinar y gestionar recursos de rehabilitación psicosocial y laboral dentro de la red pública de atención para personas con enfermedad mental. Los ámbitos de actuación de la Fundación son la rehabilitación psicosocial y laboral, los programas de apoyo y socialización, los programas residenciales, las alternativas de integración laboral, la coordinación de distintas iniciativas y programas de ámbito asociativo y el desarrollo de actividades de formación e investigación. Los patronos son las Consejerías de Sanidad, Bienestar Social, Trabajo y Empleo de la Junta de Comunidades de Castilla-La Mancha, el Servicio de Salud de Castilla-La Mancha (SESCAM) y la Federación de asociaciones de familiares y personas con enfermedad mental de Castilla-La Mancha (FEAFES C-LM). En la tabla 3 están los recursos de salud mental de los que dispone en el año 2007.

Tabla 3

Programas para personas con enfermedad mental de la Fundación FISLEM

\begin{tabular}{lcc}
\hline & N. $^{\circ}$ de dispositivos & Plazas \\
\hline $\begin{array}{l}\text { Programa de rehabilitación } \\
\text { psicosocial y laboral }\end{array}$ & 19 & \\
- Centros de Rehabilitación & 16 & 1.045 \\
$\quad$ Psicosocial y Laboral & & 240 \\
- Programa PRIS & 26 & \\
\hline Programa residencial & 2 & 139 \\
- Viviendas supervisadas & & 68 \\
- Residencias & 9 & 213 \\
\hline Programa de integración laboral & 12 & 110 \\
- Talleres Prelaborales & 6 & 73 contratos \\
- Cursos de formación profesional & 5 & 114 contratos \\
- Centros especiales de empleo & & 400 \\
- Programa de empleo con apoyo & & \\
\hline Programa de ocio y tiempo libre &
\end{tabular}

En previsión de crear 6 residencias con 240 plazas más.

El programa PRIS y de empleo con apoyo son número de profesionales.

Desde el marco de la Fundación se pretende disponer de un abanico de alternativas que van desde tratamientos rehabilitadores hasta dispositivos de 
DEBATES E INFORMES

apoyo social. Después de 5 años, FISLEM se ha consolidado como una red de recursos socio-sanitarios integrados en el servicio público de salud mental, con una oferta específica de programas para las personas con enfermedad mental y sus familias. Su corresponsabilidad pública permite que cualquier castellanomanchego con una enfermedad mental acceda al recurso que mejor se adapte a sus necesidades.

\section{Objetivos}

Con el objetivo de proveer evidencias para la planificación, necesitamos muestras reales que permitan estudiar a los pacientes que reciben la atención en la práctica diaria. Con este objetivo se desarrolló el estudio del programa PRIS. Además, de cara a valorar su funcionamiento y posibles resultados nos interesaba conocer la adecuación entre el tipo de intervención que estábamos ofreciendo y las necesidades de la población, la opinión de los profesionales de la red y de los usuarios sobre la atención prestada, así como algunas medidas que permitieran conocer la incidencia o efectividad de la intervención en la evolución psicosocial de los pacientes. Así, el estudio pretende ofrecer una descripción del perfil de los usuarios del programa, los niveles de satisfacción con la atención y el apoyo percibido, las necesidades psicosociales y el uso de recursos asociados a la patología, la calidad de vida y el deterioro o discapacidad. Por otro lado, también se incluyó una comparación pre-post para evaluar el cambio conseguido tras la intervención del programa, en una parte de las medidas estudiadas (deterioro y funcionamiento psicosocial). Se incluyeron 100 sujetos de los que se pudieron estudiar 89. La muestra se obtuvo de todas las provincias de Castilla-La Mancha. Fueron incluidos los sujetos con una enfermedad mental grave y de larga evolución, que viven en la comunidad y que reciben la atención en la red castellano-manchega. Esto incluye a pacientes con distintos grados de afectación, años de evolución y deterioro asociado.

\section{Método}

Sujetos

La población seleccionada fueron los sujetos atendidos por el programa PRIS en el año 2006 y que hubieran recibido atención durante los años 2004 y 2005, durante un mínimo de 1 año continuado. De todos los sujetos posibles se 
seleccionaron los que cumplían con los siguientes criterios de inclusión: tener un diagnóstico de enfermedad mental severa de larga evolución; residir en alguna de las áreas sanitarias de Castilla-La Mancha; tener entre 18 y 65 años; estar en tratamiento por una Unidad de Salud Mental de la región; haber tenido algún contacto con los dispositivos de salud mental de referencia en los dos años escogidos para el estudio; tener diseñado un plan individualizado de atención y seguimiento integrado dentro de un CRPSL; coordinación con los profesionales del CRPSL y la USM para el seguimiento del caso. De enero a mayo de 2006 se seleccionaron 100 sujetos. Sin embargo, de la depuración de la muestra quedaron finalmente 89. Esta modificación viene explicada por una parte de tener en cuenta únicamente los pacientes atendidos entre el año 2004 y 2005, debido a que durante ese periodo se produjo la fase de integración del programa en los CRPSL, especializando sus funciones de rehabilitación psicosocial y asegurando una continuidad en la atención (ver tabla 1). Durante el año 2002 y 2003 la movilidad de pacientes como consecuencia del ajuste de perfiles y del desarrollo del programa impide considerar parte de los sujetos incluidos inicialmente. Por otra parte, la reducción de la muestra también se explica de excluir algunos pacientes que no llegaban a 1 año de intervención. De esta forma la muestra a estudiar aunque se ve reducida presenta menos diferencias de distribución en las variables clínicas, sociales, demográficas y de procedencia o derivación, con relación al total de usuarios atendidos por el programa desde su inicio. En la tabla 4 se muestran los sujetos participantes en el estudio de cada provincia. En la tabla 5 se describen las características generales de la muestra en las variables estudiadas.

Tabla 4

Características generales de la muestra que participó en el estudio

\begin{tabular}{lc}
\hline N. ${ }^{\text {Total }}$ & 89 \\
\hline Provincia & \\
- Albacete & 25 \\
- Ciudad Real & 18 \\
- Cuenca & 10 \\
- Guadalajara & 10 \\
- Toledo & 26 \\
\hline mujeres & $66 \%$ \\
$\%$ hombres & $34 \%$ \\
\hline Media de edad & 41 \\
\hline Media de años de evolución de la enfermedad & 15,7 \\
\hline
\end{tabular}


Tabla 5

Resumen del perfil de los participantes en el estudio del programa PRIS

\begin{tabular}{ll}
\hline Minusvalía & $81(91): 8(9)$ \\
Si:No (\%) & $65.5(13.1)$ \\
Media edad del cuidador (D.T.) & $29(38 \%)$ \\
Cuidadores con >70 años & $85(95.4)$ \\
Situación laboral (\%) & $4(4.6)$ \\
Sin trabajo & $26(30)$ \\
Trabajando & $24.3(8.5)$ \\
\hline Número de hospitalizados 2 años antes (\%) & $($ rango 0 a 5) \\
\hline Media meses de atención en el programa (D.T.) & $2.5(1.5)$ \\
\hline Deterioro psicosocial (media; D.T.) & $3.5(1)$ \\
DAS-cuidado personal & $3.1(1.1)$ \\
DAS-ocupación & $3.7(1)$ \\
DAS- familia/hogar & (rango 11 a 55) \\
DAS-funcionamiento social & $48(5.8)$ \\
\hline ASP (media; D.T.) & (rango 15 a 105) \\
\hline CV (media; D.T.) & $74(15)$ \\
\hline Satisfacción de la familia (media; D.T.) & (rango 0 a 10) \\
Con la atención suministrada al usuario & $9(1.39)$ \\
Con la atención suministrada al cuidador & $9(1.2)$ \\
\hline
\end{tabular}

DAS: Escala breve de evaluación de discapacidad, ASP: Apoyo social percibido, CV: calidad de vida, DT: desviación típica.

\section{Medidas}

Con el objetivo de cuantificar las variables se elaboró una batería de instrumentos, unos de elaboración propia y otros validados y utilizados con los mismos fines en el contexto nacional o internacional. Se describen a continuación todos los instrumentos utilizados en el estudio, aunque en el presente artículo solo se presentan los resultados resumidos de algunos de ellos.

- Registro de datos personales y clínicos del usuario.

Recoge el perfil de usuarios del programa.

- Escala de necesidades. NCA (27).

Recoge la presencia o ausencia de un problema en 3 áreas: clínica, social y apoyos. En una segunda parte recoge si las necesidades están o no cubiertas. Se ha utilizado con muestras españolas similares (28). 
- Escala de características de la intervención. ECI.

Desarrollo propio. Describe el tipo de intervención que se lleva a cabo con los usuarios del programa.

- Cuestionario de satisfacción de usuarios.

Es una adaptación de la versión publicada por Cuevas y otros (29). Evalúa la opinión del usuario sobre los servicios recibidos en un sentido amplio (eficacia, satisfacción, utilidad, accesibilidad, etc.).

- Cuestionario de satisfacción de familias.

Elaboración propia. Similar al anterior pero adaptado para las familias.

- Cuestionario de opinión de los profesionales.

Elaboración propia y adaptado de Gómez Beneyto y otros (30). Recoge la opinión de otros profesionales en relación al estilo de atención de la intervención (normalización, coordinación, continuidad de cuidados, etc.).

- Escala de funcionamiento psicosocial. DAS (31).

Escala de la Organización Mundial de la Salud (OMS) que recoge discapacidad asociada al trastorno mental en cuatro áreas: autocuidados, ocupación, familia y red social.

- Cuestionario de calidad de vida. QOL.

Escala de Baker e Itagliata (32). Evalúa la satisfacción de usuario en cuanto a su hogar, salud, vestuario, amigos, ocio y otras áreas vitales.

- Escala de apoyo social percibido. DUKE-UNC.

Validada por Bellón y otros (33). Evalúa la percepción subjetiva del apoyo percibido por el usuario en relación al programa de intervención.

\section{Procedimiento}

Los datos se recogieron en el trascurso de la práctica diaria de los equipos y el procedimiento fue el siguiente: a) se entrenó a los profesionales en el uso de los instrumentos y se acordó la manera de aplicarlos y las fechas de presentación en el departamento de programas de FISLEM; b) se seleccionaron responsables de referencia para resolver dudas de los equipos en la fase de trabajo de campo; c) la recogida de información se realizó teniendo en cuenta los criterios de la tabla 6 durante los meses de febrero a mayo de 2006 y d) una vez recibidos los cuestionarios en los servicios centrales de FISLEM la información de cada uno de ellos fue depurada, verificada y analizada. 
Tabla 6

Recogida de los datos

\begin{tabular}{lll}
\hline & \multicolumn{1}{c}{ Momento } & \multicolumn{1}{c}{ Responsable } \\
\hline Información del perfil & Al comienzo del estudio & Profesional PRIS \\
\hline Revisión de historia clínica & Al comienzo del estudio & Profesional PRIS \\
\hline NCA & Al comienzo del estudio & Profesional PRIS \\
\hline ECI & Al comienzo del estudio & Profesional PRIS \\
\hline Opinión de profesionales & Al comienzo del estudio & Profesional CRPSL \\
\hline Opinión de pacientes & Al comienzo del estudio & Profesional CRPSL \\
\hline Opinión de cuidadores & Al comienzo del estudio & Profesional CRPSL \\
\hline Apoyo social & Al comienzo del estudio & Profesional PRIS \\
\hline Calidad de Vida & Al comienzo del estudio & Profesional PRIS \\
\hline Discapacidad & Pre-post & Profesional PRIS \\
\hline
\end{tabular}

Para hacer una definición operativa de las características de la intervención, se pidió a cada equipo que contestara a un cuestionario (ECI) acerca de cuáles eran los componentes críticos que definen su intervención, con el objetivo de comprobar tanto aspectos comunes como la variabilidad de la intervención.

\section{Análisis de los datos}

Se realizó el análisis de los datos con el paquete estadístico SPSS 12.1. Dichos análisis fueron exploratorios y con la intención de generar hipótesis posteriores. Algunos análisis incluyen análisis descriptivos, para analizar las características del perfil clínico y sociodemográfico. Se usaron medidas de asociación para variables distribuidas normalmente usando la correlación de Pearson. Las diferencias entre grupos se midieron usando t de Student y Anova, o el test de Kruskall Wallis y U. de Man Withney en el caso de que las variables no siguieran una distribución normal o fuesen necesarias pruebas estadísticas no paramétricas. Las variables categoriales fueron analizadas usando la prueba de Ji cuadrado mediante tablas de contingencia y utilizando los estadísticos de Pearson o Tau-b de Kendall según se tratara de variables nominales u ordinales. Para las comparaciones pre y post se utilizó la prueba de Wilcoxon.

Los datos ofrecen la posibilidad de hacer una mezcla de análisis exploratorios, ya que las medidas utilizadas tienen una mezcla de variables con diferentes niveles de medida. Por eso los análisis se limitaron según el interés de obtener un tipo determinado de información que permitiera acercarse a los datos de una mera eminentemente exploratoria. 


\section{Resultados}

A continuación se presentan parte de los resultados obtenidos principalmente en relación a las características de la intervención, el perfil de la muestra y la opinión sobre la intervención. Se trata de los resultados preliminares. En series de artículos posteriores se expondrán los resultados de cada variable estudiada en mayor profundidad.

\section{A. Análisis de las variables de la muestra y comparaciones intragrupo}

El 71,6\% de la muestra tenía un diagnóstico de esquizofrenia, el 11,4\% de Trastorno bipolar y el $8 \%$ de Trastorno de la Personalidad. El tiempo medio de atención en el programa PRIS fue de 24 meses. No se han encontrado diferencias significativas entre hombres y mujeres en edad $(\mathrm{H}=40 \mathrm{vs} . \mathrm{M}=42 ; \mathrm{t}=1.17, \mathrm{P}=0.24) \mathrm{ni}$ en años de evolución $(\mathrm{H}=14.6$ vs $\mathrm{M}=17,4 ; \mathrm{t}=1.52, \mathrm{P}=0.13)$. Tampoco se encontraron relaciones significativas entre la variable sexo y haber tenido ingresos psiquiátricos previos (tabla de contingencia $2 \times 2$ : $\mathrm{c}^{2}=0.49$, g.l $=1, \mathrm{p}=0.48$ ), ni en el número total de ingresos psiquiátricos previos a la intervención en el programa $(\mathrm{H}=3.2$ vs. $\mathrm{M}=4.5 ; \mathrm{t}=1.53, \mathrm{p}=0.12)$. Sin embargo hay una tendencia, aunque no significativa en las mujeres atendidas a tener mayor edad, mayor evolución de la enfermedad y mayor número de ingresos psiquiátricos totales. Se comprobó si existía alguna diferencia entre los años de evolución y el número de ingresos psiquiátricos los dos años antes, no encontrando diferencias significativas $\left(c^{2}=0.76, p=0.9\right)$. Tampoco se encontraron diferencias con los análisis de varianza en esta variable (ingresos 2 años antes) en relación a vivir en un contexto rural, urbano o semirural $(\mathrm{F}=024, \mathrm{P}=0.78)$.

El tipo de seguimiento clínico que se hacía desde la USM de referencia era únicamente farmacológico para el $90 \%$ de los casos, farmacológico y psicológico para el $9 \%$ de los casos y ninguno para el 1\%. El tipo de tratamiento rehabilitador que predominaba era el entrenamiento en habilidades de la vida diaria (para un $45.3 \%)$, seguido de la integración comunitaria (50\%).

\section{B. Perfil de Necesidades}

Los problemas clínicos que se presentan más frecuentemente son los síntomas psicóticos negativos (lentitud y baja actividad en el 69\% de la muestra), los síntomas psicóticos positivos (40\%) y los síntomas asociados de ansiedad (40\%). Con muy baja frecuencia se presentan problemas relacionados con el consumo de drogas $(6 \%)$. En la sección de destrezas y habilidades los problemas alcanzan una frecuencia más alta que en la sección de síntomas y comportamiento. Los problemas más frecuentes son los relacionados con el ocio $(87.5 \%)$, mantenimiento del hogar $(69 \%)$, resolución de gestiones propias $(63 \%)$, y capacidades laborales (capacidad $79 \%$ y motivación $71 \%$ ). El problema más frecuente en relación con el área de soportes es la ausencia de redes de apoyo social (80\%). 
Tabla 7

Distribución de la muestra según problemas

\begin{tabular}{|c|c|c|c|c|c|c|}
\hline \multirow{2}{*}{$\begin{array}{c}\text { SÍNTOMAS } \\
\text { ÁREAS }\end{array}$} & \multicolumn{2}{|c|}{ SIN PROBLEMA } & \multicolumn{2}{|c|}{$\begin{array}{l}\text { CON PROBLEMA } \\
\text { actual o reciente }\end{array}$} & \multicolumn{2}{|c|}{ N. ${ }^{\circ}$ Total } \\
\hline & $\mathrm{N}$ & $\%$ & $\mathrm{~N}$ & $\%$ & $\mathrm{~N}$ & $\%$ \\
\hline \multicolumn{7}{|l|}{ Síntomas y comportamientos } \\
\hline Síntomas psicóticos positivos & 53 & 60 & 35 & 40 & 88 & 100 \\
\hline Lentitud y baja actividad & 27 & 31 & 60 & 69 & 87 & 100 \\
\hline $\begin{array}{l}\text { Efectos secundarios de } \\
\text { la medicación }\end{array}$ & 69 & 79 & 18 & 21 & 87 & 100 \\
\hline $\begin{array}{l}\text { Síntomas neuróticos/ } \\
\text { de ansiedad }\end{array}$ & 53 & 60 & 36 & 40 & 89 & 100 \\
\hline Demencia o psicosis orgánica & 88 & 99 & 1 & 1 & 89 & 100 \\
\hline Síntomas físicos o enfermedades & 70 & 79 & 19 & 21 & 89 & 100 \\
\hline $\begin{array}{l}\text { Comportamiento peligroso } \\
\text { o destructivo }\end{array}$ & 77 & 87.5 & 11 & 12.5 & 89 & 100 \\
\hline $\begin{array}{l}\text { Comportamiento socialmente } \\
\text { inadecuado }\end{array}$ & 71 & 80 & 18 & 20 & 89 & 100 \\
\hline Uso de alcohol & 80 & 90 & 9 & 10 & 89 & 100 \\
\hline Uso de drogas & 84 & 94 & 5 & 6 & 89 & 100 \\
\hline \multicolumn{7}{|l|}{ Destrezas y habilidades sociales } \\
\hline Higiene personal & 43 & 49 & 45 & 51 & 88 & 100 \\
\hline Vestido & 53 & 61 & 34 & 39 & 87 & 100 \\
\hline Cuidados de la salud general & 47 & 53 & 41 & 47 & 88 & 100 \\
\hline Alimentación & 51 & 61 & 33 & 39 & 84 & 100 \\
\hline Estructuración del sueño & 45 & 51 & 44 & 49 & 89 & 100 \\
\hline Tareas domésticas & 26 & 31 & 57 & 69 & 83 & 100 \\
\hline Manejo del dinero & 36 & 40 & 53 & 60 & 89 & 100 \\
\hline Uso del tiempo de ocio & 11 & 12.5 & 77 & 87.5 & 88 & 100 \\
\hline Manejo de transportes & 63 & 72 & 25 & 28 & 88 & 100 \\
\hline Habilidades de comunicación & 39 & 44 & 49 & 56 & 88 & 100 \\
\hline Capacidad cognitiva (atención) & 45 & 52 & 42 & 48 & 87 & 100 \\
\hline $\begin{array}{l}\text { Resolución de gestiones } \\
\text { propias }\end{array}$ & 31 & 36 & 56 & 63 & 89 & 100 \\
\hline $\begin{array}{l}\text { Responsabilidad ante su } \\
\text { tratamiento }\end{array}$ & 45 & 51 & 43 & 49 & 88 & 100 \\
\hline Capacidad laboral & 18 & 21 & 69 & 79 & 87 & 100 \\
\hline Motivación laboral & 25 & 29 & 60 & 71 & 85 & 100 \\
\hline \multicolumn{7}{|l|}{ Soportes sociales } \\
\hline Redes de apoyo social & 17 & 19.5 & 70 & 80 & 87 & 100 \\
\hline Apoyo familiar & 47 & 54 & 40 & 46 & 87 & 100 \\
\hline Soporte residencial & 67 & 81 & 16 & 19 & 83 & 100 \\
\hline
\end{tabular}


Tabla 8

Distribución de la muestra según estado de las necesidades

\begin{tabular}{|c|c|c|c|c|c|c|}
\hline \multirow{2}{*}{$\begin{array}{c}\text { SÍNTOMAS } \\
\text { ÁREAS } \\
\end{array}$} & \multicolumn{2}{|c|}{ CUBIERTAS } & \multicolumn{2}{|c|}{$\begin{array}{l}\text { NO } \\
\text { CUBIERTAS }\end{array}$} & \multicolumn{2}{|c|}{$\begin{array}{c}\text { SIN } \\
\text { POSIBILIDAD } \\
\text { DE CUBRIR }\end{array}$} \\
\hline & $\mathrm{N}$ & $\%$ & $\mathrm{~N}$ & $\%$ & $\mathrm{~N}$ & $\%$ \\
\hline \multicolumn{7}{|l|}{ Síntomas y comportamientos } \\
\hline Síntomas psicóticos positivos & 31 & 86 & 3 & 8 & 2 & 6 \\
\hline Lentitud y baja actividad & 36 & 61 & 19 & 32 & 4 & 7 \\
\hline $\begin{array}{l}\text { Efectos secundarios de } \\
\text { la medicación }\end{array}$ & 13 & 68 & 5 & 26 & 1 & 5 \\
\hline $\begin{array}{l}\text { Síntomas neuróticos/ } \\
\text { de ansiedad }\end{array}$ & 27 & 73 & 7 & 19 & 3 & 8 \\
\hline Demencia o psicosis orgánica & 1 & 100 & - & - & - & - \\
\hline Síntomas físicos o enfermedades & 14 & 70 & 5 & 25 & 1 & 5 \\
\hline $\begin{array}{l}\text { Comportamiento peligroso } \\
\text { o destructivo }\end{array}$ & 11 & 79 & 2 & 14 & 1 & 7 \\
\hline $\begin{array}{l}\text { Comportamiento socialmente } \\
\text { inadecuado }\end{array}$ & 14 & 70 & 4 & 20 & 2 & 10 \\
\hline Uso de alcohol & 6 & 60 & 4 & 40 & - & 0 \\
\hline Uso de drogas & 5 & 100 & - & - & - & - \\
\hline \multicolumn{7}{|l|}{ Destrezas y habilidades sociales } \\
\hline Higiene personal & 35 & 81 & 5 & 12 & 3 & 7 \\
\hline Vestido & 33 & 90 & 2 & 5 & 2 & 5 \\
\hline Cuidados de la salud general & 37 & 86 & 3 & 7 & 3 & 7 \\
\hline Alimentación & 24 & 80 & 7 & 16 & 2 & 4 \\
\hline Estructuración del sueño & 33 & 75 & 8 & 18 & 3 & 7 \\
\hline Tareas domésticas & 42 & 76 & 12 & 19 & 3 & 5 \\
\hline Manejo del dinero & 40 & 78 & 10 & 20 & 1 & 2 \\
\hline Uso del tiempo de ocio & 36 & 50.5 & 29 & 41 & 6 & 8.5 \\
\hline Manejo de transportes & 18 & 68 & 9 & 33 & - & - \\
\hline Habilidades de comunicación & 41 & 82 & 7 & 14 & 2 & 4 \\
\hline Capacidad cognitiva (atención) & 32 & 74 & 8 & 19 & 3 & 7 \\
\hline $\begin{array}{l}\text { Resolución de gestiones } \\
\text { propias }\end{array}$ & 47 & 89 & 3 & 6 & 3 & 6 \\
\hline $\begin{array}{l}\text { Responsabilidad ante su } \\
\text { tratamiento }\end{array}$ & 40 & 83 & 3 & 6 & 5 & 10 \\
\hline Capacidad laboral & 27 & 40 & 31 & 46 & 9 & 13 \\
\hline Motivación laboral & 26 & 42 & 25 & 40 & 11 & 18 \\
\hline \multicolumn{7}{|l|}{ Soportes sociales } \\
\hline Redes de apoyo social & 26 & 41 & 29 & 45 & 9 & 14 \\
\hline Apoyo familiar & 27 & 62 & 11 & 26 & 5 & 12 \\
\hline Soporte residencial & 6 & 37.5 & 6 & 37.5 & 4 & 25 \\
\hline
\end{tabular}


Tabla 9

Distribución del número de problemas por áreas

\begin{tabular}{lrrrrrr}
\hline \multicolumn{1}{c}{ ÁREAS } & \multicolumn{2}{c}{ CUBIERTAS } & \multicolumn{2}{c}{ NO CUBIERTAS } & \multicolumn{2}{c}{$\begin{array}{c}\text { SIN POSIBILIDAD } \\
\text { DE CUBRIRSE }\end{array}$} \\
\hline & $\mathrm{n}$ & $\%$ & $\mathrm{n}$ & $\%$ & $\mathrm{n}$ & $\%$ \\
\hline Síntomas $(\mathrm{n}=212)$ & 158 & 75 & 49 & 20 & 14 & 5 \\
\hline Habilidades $(\mathrm{n}=728)$ & 511 & 70 & 162 & 22 & 56 & 8 \\
\hline Soportes $(\mathrm{n}=126)$ & 59 & 47 & 46 & 36 & 18 & 17 \\
\hline
\end{tabular}

Dentro de los problemas clínicos los síntomas psicóticos y de drogas son los que se encuentran más frecuentemente cubiertos (86\% y $100 \%$ respectivamente), mientras que los síntomas negativos, los problemas de alcohol y los efectos secundarios lo están en menor medida (porcentajes no cubiertos del 32\%, 40\%, 26\%). En el área de habilidades y destrezas los problemas están cubiertos en menor frecuencia que en la sección clínica, demostrándose esta área como más deficitaria en el perfil de necesidad de los pacientes estudiados. Los problemas relacionados con higiene personal (81\%), vestido (90\%), alimentación (80\%) están más cubiertos en comparación con los de ocupación, trabajo, dinero y tareas domésticas que son las que están más frecuentemente no cubiertas. En el área de soportes se encuentran los mayores problemas y están menos cubiertos que los síntomas clínicos y las habilidades. Los problemas de esta área menos cubiertos por orden de importancia son los de la red de apoyo, el soporte residencial y el apoyo familiar. Respecto al estado de necesidad (tabla 9), la mayoría de los problemas clínicos están cubiertos (75\%), en el área de habilidades este porcentaje disminuye (70\%). En el área de soportes se encuentran el mayor porcentaje de problemas sin cubrir (36\%). Pocos problemas se presentan sin posibilidad de cubrirse, sin embargo en el área de soportes el $17 \%$ de ellos no tienen posibilidad de cubrirse con los recursos disponibles en la red en el momento del estudio.

\section{Características de la intervención}

La intervención se acerca en gran medida al marco teórico y metodológico de referencia en la ratio profesional: usuarios (10-15 para el 54\% de los equipos), intervención compartida con el equipo de CRPSL $(84,6 \%)$, frecuencia de contactos semanal con el usuario (54\%) y en la comunidad (50\%) o el domicilio (50\%), y frecuencia de contactos con la familia quincenal (69\%). Las principales demandas de intervención realizadas al programa por orden de 
importancia son: el enganche al CRPSL, evitar la desvinculación de la red, acompañamientos y apoyo social-afectivo. Un apartado importante es la movilidad de los profesionales en el último año $(54,5 \%)$, aspecto que se ha relacionado con malos resultados para este tipo de programas en algunas investigaciones (34). No se encontraron relaciones significativas entre el número de pacientes asignados a cada profesional y la frecuencia de contactos con el usuario $\left(\mathrm{c}^{2}\right.$ de Pearson $\left.=1.408 ; \mathrm{p}=0.7\right)$, ni con la frecuencia de contactos con la familia $\left(c^{2}\right.$ de Pearson $\left.=2.94 ; p=0.4\right)$. Tampoco se encontró relación entre la frecuencia de contacto con el usuario y con la familia $(\mathrm{P}=0.47)$.

Tabla 10

Resumen de la escala de características de la intervención

\begin{tabular}{|c|c|c|c|c|c|c|}
\hline $\begin{array}{l}\text { CARACTERÍSTICAS } \\
\text { DE LA } \\
\text { INTERVENCIÓN }\end{array}$ & OPCIONES & & & $\%$ & & \\
\hline \multirow[t]{2}{*}{$\begin{array}{l}\text { Ratio profesional } \\
\text { /usuarios }\end{array}$} & $\begin{array}{l}\square 10-15 \\
\square 16-20 \\
\square 21-25 \\
\square>25\end{array}$ & & & $\begin{array}{r}53,8 \\
15,4 \\
7,7 \\
23,1\end{array}$ & & \\
\hline & & Nunca & Ocasional & $\begin{array}{c}\text { A } \\
\text { menudo }\end{array}$ & $\begin{array}{c}\text { Con casi } \\
\text { todos }\end{array}$ & $\begin{array}{l}\text { Con } \\
\text { todos }\end{array}$ \\
\hline \multirow{7}{*}{$\begin{array}{l}\text { Tipo de intervención } \\
\text { realizada: }\end{array}$} & Enganche al CRPSL: & 0 & 0 & 23,1 & 69,2 & 7,7 \\
\hline & $\begin{array}{l}\text { Evitar desvinculación de } \\
\text { la red socio-sanitaria: }\end{array}$ & 0 & 7,7 & 15,4 & 53,8 & 23,1 \\
\hline & Apoyo social-afectivo: & 0 & 15,4 & 23,1 & 30,8 & 30,8 \\
\hline & Accesibilidad: & 15,4 & 7,7 & 38,5 & 15,4 & 23,1 \\
\hline & Integración laboral: & 23,1 & 38,5 & 38,5 & 0 & 0 \\
\hline & $\begin{array}{l}\text { Acompañamiento a } \\
\text { recursos y gestiones: }\end{array}$ & 0 & 7,7 & 53,8 & 23,1 & 15,4 \\
\hline & Orientación: & 0 & 7,7 & 30,8 & 30,8 & 30,8 \\
\hline $\begin{array}{l}\text { Intervención con } \\
\text { los casos } \\
\text { compartida equipo } \\
\text { del CRPSL }\end{array}$ & $\begin{array}{l}\square \text { Sí } \\
\square \text { NO }\end{array}$ & & & $\begin{array}{l}84,6 \\
15,4\end{array}$ & & \\
\hline $\begin{array}{l}\text { Participación del } \\
\text { usuario en la toma } \\
\text { de decisiones }\end{array}$ & $\begin{array}{l}\text { - Alta } \\
\text { - Moderada } \\
\text { - Baja } \\
\text { - No }\end{array}$ & & & $\begin{array}{c}69,2 \\
23,1 \\
7,7 \\
0\end{array}$ & & \\
\hline $\begin{array}{l}\text { Énfasis en el } \\
\text { entrenamiento } \\
\text { en habilidades }\end{array}$ & $\begin{array}{l}\text { - Alta } \\
\text { - Moderada } \\
\text { - Baja } \\
\text { - No }\end{array}$ & & & $\begin{array}{c}46,2 \\
53,8 \\
0 \\
0\end{array}$ & & \\
\hline
\end{tabular}


Tabla 10

Resumen de la escala de características de la intervención (Continuación)

\begin{tabular}{|c|c|c|}
\hline $\begin{array}{l}\text { CARACTERÍSTICAS } \\
\text { DE LA } \\
\text { INTERVENCION }\end{array}$ & OPCIONES & $\%$ \\
\hline $\begin{array}{l}\text { Frecuencia de } \\
\text { contactos con } \\
\text { el usuario }\end{array}$ & $\begin{array}{l}\text { - Semanal } \\
\text { - Quincenal } \\
\text { - Mensual } \\
\text { - > } 1 \text { vez al mes }\end{array}$ & $\begin{array}{c}53,8 \\
46,2 \\
0 \\
0 \\
\end{array}$ \\
\hline $\begin{array}{l}\text { Lugar principal } \\
\text { de los contactos }\end{array}$ & $\begin{array}{l}\text { - Centro } \\
\text { - Comunidad } \\
\text { - Domicilio }\end{array}$ & $\begin{array}{r}0 \\
50 \\
50\end{array}$ \\
\hline $\begin{array}{l}\text { Intervención } \\
\text { integrada en } \\
\text { Plan del CRPSL }\end{array}$ & $\begin{array}{l}\text { - Con todos los casos } \\
\text { - Con ningún caso } \\
\text { - Con algún caso }\end{array}$ & $\begin{array}{r}100 \\
0 \\
0\end{array}$ \\
\hline $\begin{array}{l}\text { Carga afectiva } \\
\text { de los lazos } \\
\text { entre el profesional } \\
\text { y el usuario }\end{array}$ & $\begin{array}{l}\text { - Débil } \\
\text { - Moderada } \\
\text { - Fuerte }\end{array}$ & $\begin{array}{c}15,4 \\
84,6 \\
0\end{array}$ \\
\hline $\begin{array}{l}\text { Vinculación con } \\
\text { recursos } \\
\text { comunitarios }\end{array}$ & $\begin{array}{l}\text { - Ninguna } \\
\text { - Baja } \\
\text { - Moderada } \\
\text { - Alta } \\
\end{array}$ & $\begin{array}{c}0 \\
0 \\
58,3 \\
41,7 \\
\end{array}$ \\
\hline $\begin{array}{l}\text { Intervención con } \\
\text { familias y otros } \\
\text { miembros cuidadores }\end{array}$ & $\begin{array}{l}\text { - Ninguna } \\
\text { - Baja } \\
\text { - Moderada } \\
\text { - Alta }\end{array}$ & $\begin{array}{c}0 \\
0 \\
61,5 \\
38,5\end{array}$ \\
\hline $\begin{array}{l}\text { Frecuencia de } \\
\text { contactos con } \\
\text { la familia }\end{array}$ & $\begin{array}{l}\text { - Semanal } \\
\text { - Quincenal } \\
\text { - Mensual } \\
\text { - > } 1 \text { vez al mes }\end{array}$ & $\begin{array}{c}15,4 \\
69,2 \\
15,4 \\
0\end{array}$ \\
\hline $\begin{array}{l}\text { Existencia de } \\
\text { reuniones con } \\
\text { el equipo del } \\
\text { CRPSL para } \\
\text { revisar los casos }\end{array}$ & $\begin{array}{l}\text { - Semanal } \\
\text { - Quincenal } \\
\text { - Mensual } \\
\text { - > } 1 \text { vez al mes } \\
\text { - NO }\end{array}$ & $\begin{array}{c}66,7 \\
16,7 \\
16,6 \\
0 \\
0 \\
\end{array}$ \\
\hline $\begin{array}{l}\text { Movilidad del } \\
\text { equipo en } \\
\text { el último año }\end{array}$ & $\begin{array}{l}\square \text { SÍ } \\
\square \text { NO }\end{array}$ & $\begin{array}{l}54,5 \\
45,5\end{array}$ \\
\hline Perfil del paciente & $\begin{array}{l}\text { - Hay criterios explícitos } \\
\text { de adscripción al PRIS } \\
\text { - No hay criterios explícitos } \\
\text { de adscripción al PRIS }\end{array}$ & $\begin{array}{r}100 \\
0\end{array}$ \\
\hline $\begin{array}{l}\text { Duración de } \\
\text { la intervención }\end{array}$ & $\begin{array}{l}\text { - Limitada } \\
\text { - Ilimitada }\end{array}$ & $\begin{array}{l}50 \\
50\end{array}$ \\
\hline $\begin{array}{l}\text { Criterios de } \\
\text { evaluación de } \\
\text { resultados } \\
\text { con los usuarios }\end{array}$ & - SÍ & $\begin{array}{r}91,7 \\
8,3\end{array}$ \\
\hline
\end{tabular}


D. Opinión de los pacientes sobre la intervención

El 50\% de los casos opinaba que la eficacia de la intervención para resolver sus problemas personales había sido satisfactoria o excelente, y nadie opinaba que había sido insatisfactoria. Un 83\% opinaba que la intervención había servido para ayudar a sus familiares más cercanos a conocer y comprender mejor sus problemas. El 93\% opinaba que la intervención había servido para ayudarle a mejorar el conocimiento y comprensión de sus problemas y dificultades. Destacable es también la opinión en relación a la eficacia de la ayuda recibida para entrar en contacto con los servicios normalizados de su comunidad, el 88\% de los casos afirmaba que le habían ayudado en este aspecto.

\section{E. Opinión de los cuidadores}

El 60\% de los familiares afirmaba que desde que el PRIS está interviniendo con su familiar sus salidas a la calle son más frecuentes que antes. En cuanto al nivel de actividad del paciente a lo largo del día, el $71 \%$ de los cuidadores opina que la actividad diaria ha aumentado desde que recibe las visitas del profesional. En relación a la capacidad de escucha de los profesionales, el 97\% de las familias entrevistas opinaba que cuando hablan con el profesional del PRIS pueden hablar sin prisas y que le escuchan con atención. El 100\% opina que la actitud del profesional es de amabilidad y calidez. También en relación a la utilidad de la intervención para mejorar la convivencia ha sido valorada positivamente por el $93 \%$ de los cuidadores. Ninguna familia opinó que la intervención hubiera servido para empeorar la convivencia y las relaciones con los pacientes.

F. Opinión de los profesionales de los equipos donde el programa se integró

Todos los profesionales de los centros donde el programa PRIS se integró piensan que el PRIS facilita el seguimiento de los casos y la valoración de sus necesidades en cada momento. El 85\% afirmó que el profesional facilitó o mejoró la relación del centro con los servicios sociales. El 100\% de los centros opinaba que el programa posibilitaba ampliar la atención tradicional que se daba a los pacientes crónicos en el área con otras intervenciones (apoyo social, apoyo familiar, orientación cotidiana, acompañamiento para el ocio y la ocupación). En todos los centros entrevistados contestaron que se había incrementado su satisfacción profesional con la atención prestada a los pacientes. 


\section{G. Resultados en deterioro psicosocial}

Los resultados con el test de Wilcoxon indican que el cambio producido por la intervención es estadísticamente significativo en todas las áreas de funcionamiento, reduciéndose el grado de discapacidad asociada a cada área. Se produce una reducción de la discapacidad del $40 \%$ en los autocuidados personales (higiene, vestido, hábitos de salud); una reducción del deterioro de $31 \%$ en el área de ocupación del tiempo libre; una reducción de $32 \%$ en el área de familia y hogar; y una reducción del $30 \%$ en el funcionamiento en contextos sociales. Todas las puntuaciones han bajado de una discapacidad seria-severa a una discapacidad moderada-ligera.

Tabla 11

Cambios en áreas de deterioro psicosocial al inicio de la intervención $y$ en el momento de la evaluación

\begin{tabular}{lcccc}
\hline & $\begin{array}{l}\text { L.Base (pre) } \\
\text { Media (d.t.) } \\
\text { (0 a 5) }\end{array}$ & $\begin{array}{l}\text { Momento } \\
\text { de la } \\
\text { evaluación (post*) }\end{array}$ & N & P \\
\hline DAS. Cuidado personal & $2,5(1,5)$ & $1,5(1,2)$ & 89 & $\mathrm{P}<0,001$ \\
\hline DAS Ocupación & $3,5(1)$ & $2,4(1,3)$ & 89 & $\mathrm{P}<0,001$ \\
\hline DAS Familia y hogar & $3,1(1,1)$ & $2,1(1,2)$ & 89 & $\mathrm{P}<0,001$ \\
\hline DAS Funcionamiento social & $3,7(1)$ & $2,6(1,3)$ & 89 & $\mathrm{P}<0,001$ \\
\hline
\end{tabular}

* Todos los sujetos evaluados en el momento de la investigación llevan una media de intervención de 24 meses en el programa.

\section{Conclusiones}

Las medidas de evaluación utilizadas se seleccionaron por su facilidad de administrar y que no entorpecieran la práctica de los profesionales. Aunque algunas no lleguen a tener una fiabilidad óptima este criterio, unido a la selección de pacientes representativos del programa, hace reducir o limitar el error de la varianza. Así, el presente estudio seleccionó criterios de inclusión que permitieran dar un reflejo de la práctica diaria de los profesionales en su rutina profesional y de los usuarios reales a los que atienden. Los usuarios más alejados opinan más positivamente sobre el programa que los menos alejados. Este apoyo parece responder a las características de la atención del PRIS (en el domicilio, con contactos telefónicos frecuentes, y priorizando en ayudas prácticas como puede ser el acompañamiento y la compañía para personas que carecen de red de apoyo y están más aisladas). Se han encontrado cambios 
significativos entre el antes y el después de la intervención en la reducción de las discapacidades asociadas al trastorno, que junto a las opiniones de los usuarios y cuidadores reflejan el éxito de este tipo de programas comunitarios. El estilo y características de la intervención demuestran no solo la utilidad del programa PRIS para la población de personas con enfermedad mental en estos contextos rurales, sino la adecuación del programa a los objetivos por los que se puso en marcha.

\section{BIBLIOGRAFÍA}

(1) Junta de Comunidades de Castilla-La Mancha. Consejería de Sanidad. Plan de Salud Mental de Castilla-La Mancha, 2002-2004, Toledo, 2002.

(2) Junta de Comunidades de Castilla-La Mancha. Consejería de Sanidad, Plan de Salud Mental de Castilla-La Mancha, 2005-2010, Toledo, 2006.

(3) MARIANo, H. M., «Los programas de seguimiento intensivo en la Comunidad: una alternativa a la hospitalización ¿y algo más?», en Rivas Guerrero, F. (comp), La Psicosis en la Comunidad, AEN Estudios, 2002, pp.131-189.

(4) NaVARro, B. D., «Rehabilitación Psicosocial para enfermos mentales graves en contextos rurales», Revista de la AEN, 2003, 86, pp. 105-122.

(5) Junta de Comunidades de Castilla-La Mancha. Consejería de Bienestar Social, II Plan de Integración Social de Castilla-La Mancha, 2002-2005.

(6) Alonso, M.; Bravo, M. F.; y LiRIA, A., «Origen y desarrollo de los programas de seguimiento y cuidados para pacientes mentales graves y crónicos en la comunidad», Revista de la AEN, 2004, 92, pp. 25-51.

(7) GonZÁlEZ, A., «Programas de seguimiento o continuidad de cuidados en la comunidad», Revista de la AEN, 2001, pp. 4-16.

(8) BACHRACH, L. L., «Continuity of Care and Approaches to Case Management for Long Term Mentally Ill Patients», Hospital and Community Psychiatry, 1993, 44 (5), pp. 465-468.

(9) Mueser, K. T., y otros, «Models of Community Care for Severe Mental Illness: a Review of Research on Case Management», Schizophrenia Bulletin, 1998, 24, pp. 37-74.

(10) UK 700 GROUP, «Comparison of Intensive and Standard Case Management for Patients with Psychosis», British Journal of Psychiatry, 1999, 174, pp. 74-78.

(11) Holloway, F.; CARSON, J., «Intensive Case Management for the Severely Mentally Ill», British Journal of Psychiatry, 1998, 172, pp. 19-22.

(12) BoRland, G. R., "Assertive Community Treatment for Frequent Users of Psychiatric Hospitals in a Large City: a Controlled Study», American Journal of Community Psychology, 1990, 18 , pp. 865-891.

(13) Marshall, M.; Lockwood, A., Assertive Community Treatment for People with Severe Mental Disorders, Cochrane Database Syst. Rev., 2000.

(14) Marshall, M., «Case Management for People with Severe Mental Disorders», Cochrane Database Syst. Rev., 2000.

(15) WyKES, T., «Effects of Community Services on Disability and Symptoms. PriSM Psychosis Study 4», British Journal of Psychiatry, 1998, 173, pp. 385-3902.

(16) LATIMER, E. A., «Economic Impacts of Assertive Community Treatment: a Review of the Literature», Canadian Journal of Psychiatry, 1999, 44, pp. 443-454.

(17) Mueser, K., y otros, «El tratamiento comunitario de la esquizofrenia y otros trastornos mentales severos: resultados», en LARA, L.; LÓPEZ, M., Integración laboral de personas con trastorno mental severo, 2003, pp. 26-64. 
(18) SAN EMETerio, M., «Continuidad asistencial en los circuitos psiquiátricos», en LeAL, J; EsCUDERO, A., La continuidad de cuidados y el trabajo en red en salud mental, AEN Estudios, 2006, pp. 117-130.

(19) Thornicroft, G., y otros, «From Efficacy to Effectiveness in Community Mental Health Services», British Journal of Psychiatry, 1998, 173, pp. 423-427.

(20) Thornicroft, G., y otros, «Community Mental Health Teams: Evidence or Belief?», British Journal of Psychiatry, 1999, 175, pp. 508-513.

(21) Marshall, M., y otros. «PriSM Psychosis Study. Design Limitations, Questionable Conclusions», British Journal of Psychiatry, 1999, 175, pp. 501-503.

(22) Thornicroft, G., y otros, «Rationale and Design. PriSM Psicosis Study I», British Journal of Psychiatry, 1998, 173, pp. 363-370.

(23) Santos, A. B., y otros, «Provinding Assertive Community Treatment for Severely Mentally Ill Patients in a Rural Area», Hospital and Community Psychiatry, 1993, 44 (1), pp. 34-39.

(24) GolD, B., y otros, «Randomized Trial of Supported Employment Integrated with Assertive Community Treatment for Rural Adults with Severe Mental Illness», Schizophrenia Bulletin, 2006, 32 (2), pp. 378-395.

(25) Sullivan, G.; Jackson, C. A.; Spritzer, B. A., «Characteristics and Service Use of Seriously Mentally Ill Persons Living in Rural Areas», Psychiatric Services, 1996, 47 (1), pp. 57-61.

(26) Bonynge, E. R.; LeE, R.; Thurber, S., «A Profile of Mental Health Crisis Response in a Rural Setting», Community Mental Health Journal, 2005, 41 (6), pp. 675-685.

(27) BREWIN, C. R.; WING, J. K., Manual of the MCR Needs for the Care Assessment, Londres, MRC, 1989.

(28) Moreno, B., y otros, «Evaluación de las necesidades asistenciales de una muestra de pacientes esquizofrénicos del área de salud mental Granada Sur», Actas Españolas de Psiquiatría, 31 (6), 2003, pp. 325-330.

(29) Cuevas, C., y otros, «Percepción de familiares de pacientes psicóticos sobre un servicio de rehabilitación de salud mental», Revista del Colegio de Psicólogos de Andalucía Occidental, 46, 1996, pp. 71-90.

(30) GómEZ, B. M., y otros, «Informe sobre los recursos y el funcionamiento de la atención a la salud mental en la Comunidad Autónoma de Valencia», en Verdugo, M. A., y otros (comp.), Atención comunitaria, rehabilitación y empleo, INICO, 2003, pp. 440-493.

(31) OMS, Escala de evaluación de la Discapacidad WHO-DAS, 1997.

(32) BAKER, F.; INTAGLIATA, J., «Quality of Life in the Evaluation of Community Support Systems», 1982, en Bobes, G.; Portilla, M.; García, M. B., Calidad de vida en las Esquizofrenias, J. R Prous editores, 1995.

(33) Bellón, J. A.; Delgado, A.; Luna, J.; Lardelli, P., «Validez y fiabilidad del cuestionario de apoyo social Duke-UNC», Atención Primaria, 18, 1996, pp. 153-163. Extraído del Servicio Andaluz de Salud, «Cuestionarios, test e índices para la valoración del paciente en salud mental» (2004), Consejería de Salud.

(34) MCDOnel, E. C.; Bond, G. R.; SAlyers, M., y otros, «Implementing Assertive Community Treatment Programs in Rural Areas», Administration and Policy in Mental Health, 1997, 25 (2), pp. 153-173.

* Daniel Navarro Bayón, Psicólogo. Jefe del Departamento de Programas de la Fundación FISLEM; José Antonio Contreras Nieves. Director de la Fundación FISLEM.

Correspondencia: Daniel Navarro Bayón. Fundación FISLEM. Avda. Madrid, 14 1. ${ }^{\circ}$ C 45003 Toledo dnavarro@jccm.es

** Fecha de recepción: 1-VI-2007 (aceptado el 11-IV-2008). 